EPJ Web of Conferences 41, 01016 (2013)

DOI: $10.1051 /$ epjconf/20134101016

(C) Owned by the authors, published by EDP Sciences, 2013

\title{
High Order Harmonic Generation in Three Pulse Scattering Geometry
}

\author{
Caterina Vozzi ${ }^{1}$, Matteo Negro ${ }^{2}$, Luca Poletto ${ }^{1}$, Sandro De Silvestri ${ }^{2}$, and Salvatore Stagira ${ }^{2, a}$ \\ 1 Istituto di Fotonica e Nanotecnologie - CNR, piazza L. da Vinci 32, 20133 Milano, Italy \\ 2 Dipartimento di Fisica - Politecnico di Milano, piazza L. da Vinci 32, 20133 Milano, Italy
}

\begin{abstract}
We study the generation of high order harmonics produced by the interaction of three intense and ultrafast noncollinear laser pulses. Harmonic radiation, not overlapped to the direction of the incident pulses, is observed.
\end{abstract}

\section{Introduction}

High order harmonic generation (HHG) is a well studied phenomenon with numerous implications in attosecond science and matter physics [1]. HHG is generally performed in collinear geometry; in this configuration, the extreme ultraviolet (XUV) radiation copropagates with the foundamental laser pulse inside the generating medium; hence the exploitation of high harmonics, as well as their characterization, requires the use of laser-blind detectors or the selective attenuation of the laser radiation with unavoidable reduction of the XUV energy. The collinear generation geometry also obscures the presence of tiny contributions to HHG from excited atomic or molecular species, thus hindering the exploitation of harmonic generation to the spectroscopy of matter.

A possible solution to those drawbacks is the generation of high-order harmonics in non conventional geometries. Recent studies addressed HHG by interfering laser beams in several configurations, for instance in a grating of excited population induced by low-intensity beams, where only one strong pulse generated harmonics [2]; in HHG by two noncollinear intense driving beams of different wavelengths [3] and in the case of harmonics generated by one pulse and spatially dispersed by a Bragg grating produced by two counter-propagating intense laser beams [4]. In all those studies, harmonics were observed at propagation directions not coincident with the driving pulses. Moreover the investigations reported in $[3,4]$, performed in the strong interaction regime with multiple intense beams, showed that the harmonic radiation was spectrally dispersed, namely different harmonics propagated at different directions.

In this work we report on a novel regime of HHG by multiple intense beams, where the harmonics are produced by three synchronized, intense and ultrashort laser pulses arranged in a non-planar geometry. The case we explore is well within the strong-field regime, namely each of the three pulses is able to drive HHG. However, with respect to previous studies performed in similar conditions, the XUV emission we observe is not spectrally dispersed since the phase mathing conditions are fulfilled for all the harmonic orders along the observation direction.

\section{Experimental setup and theoretical predictions}

The laser emission generated by a Ti:Sapphire laser system in chirped pulse amplification (60 fs, 120 $\mathrm{mJ}$ of maximum energy, repetition rate of $10 \mathrm{~Hz}$ ) is split in four identical beams; temporal synchronization among the beams is obtained by remotely controlled delay lines. The beams are separately

a e-mail: salvatore.stagira@fisi.polimi.it

This is an Open Access article distributed under the terms of the Creative Commons Attribution License 2.0, which permits unrestricted use, distribution, and reproduction in any medium, provided the original work is properly cited. 


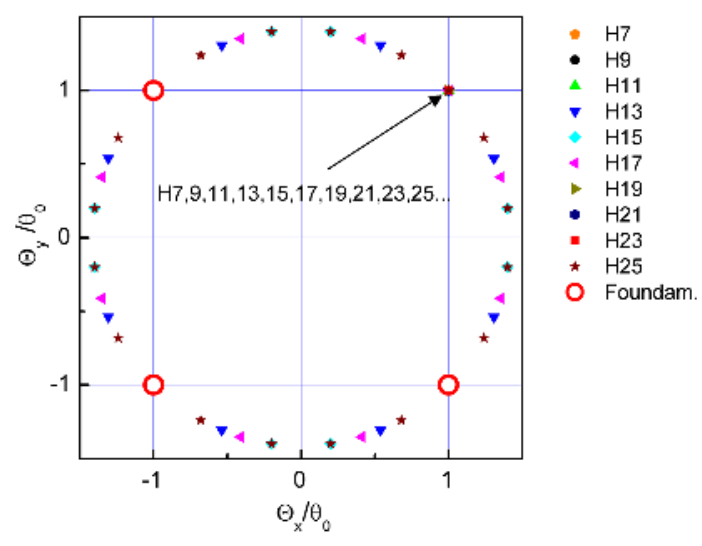

Fig. 1. Predicted emission directions of harmonics generated by three $800-\mathrm{nm}$ laser beams (shown as empty circles) positioned at the three vertices of a square, for orders ranging from 7 th to 25 th. Harmonics are not spectrally dispersed only in the direction of the forth vertex.

sent to a vacuum chamber, where they are made parallel with the beam centroids passing though the vertices of a square having $1-\mathrm{cm}$ sides. The beams are then focused in the generating medium (a pulsed gas jet operated by a valve at a backing pressure of 4 bars and synchronized to the laser pulses) by a spherical mirror with $20-\mathrm{cm}$ focal length; the estimated focal peak intensity of each of the beams is about $10^{14} \mathrm{~W} / \mathrm{cm}^{2}$. The fourth beam (hereafter called reference beam) is aligned to the entrance of a flat-field XUV spectrometer, whereas the remaining three beams are stopped; harmonics of the reference pulse are detected in the region between 16 and $36 \mathrm{eV}$. Synchronization and spatial overlap among the beams is obtained by minimizing separately the harmonic emission of the reference in presence of each of the other three beams. After completion of the beam alignment, the reference beam is blocked and harmonic emission from the interaction among the three remaining beams is detected in the direction of the (blocked) reference one. The harmonic radiation has been studied as a function of the delay between the three pulses in several gases (xenon, krypton, argon).

According to energy and momentum conservation laws [3], the directions of emitted harmonics with respect to the three driving beams should behave as reported in Fig. 1. In particular, spectrally dispersed harmonics are expected to be generated along a phase matching cone passing through the directions of the three generating foundamental beams. However, nondispersed (i.e. copropagating) harmonics are also expected along the reference direction. This prediction is confirmed by numerical simulations of high-order harmonic generation performed in the adiabatic saddle-point approximation (not shown) [5], that assign also a predominant contribution of long electron quantum paths to this emission.

\section{Experimental results and discussion}

Figure 2(a) shows the harmonics spectra measured in krypton as a function of the delay of one laser pulse with respect to the other two (which are kept synchronized); each spectrum is averaged over 50 laser shots. As one can also see from the spectral integral of the signal shown in Fig. 2(b), a clear harmonic emission is observed only when the three pulses are overlapped in time; very weak harmonics can be also detected for positive delays and are attributed to the plasma grating induced by the fixed pulses. The observation of several harmonics demostrates that harmonic emission is not spectrally dispersed as reported in other studies $[3,4]$. The peak position of the harmonics as well as their width slightly change with the delay; such behavior, that is still under investigation, could be attributed to the effect of ionization or to volume effects related to the spatio-temporal evolution of the interference among the beams in the gaseous medium. Similar results were observed in argon (not shown) and xenon. Figure 2(c) shows a comparison between harmonic spectra observed in krypton and xenon at zero delays; in this case the spectra are averaged over 500 shots in order to improve the 

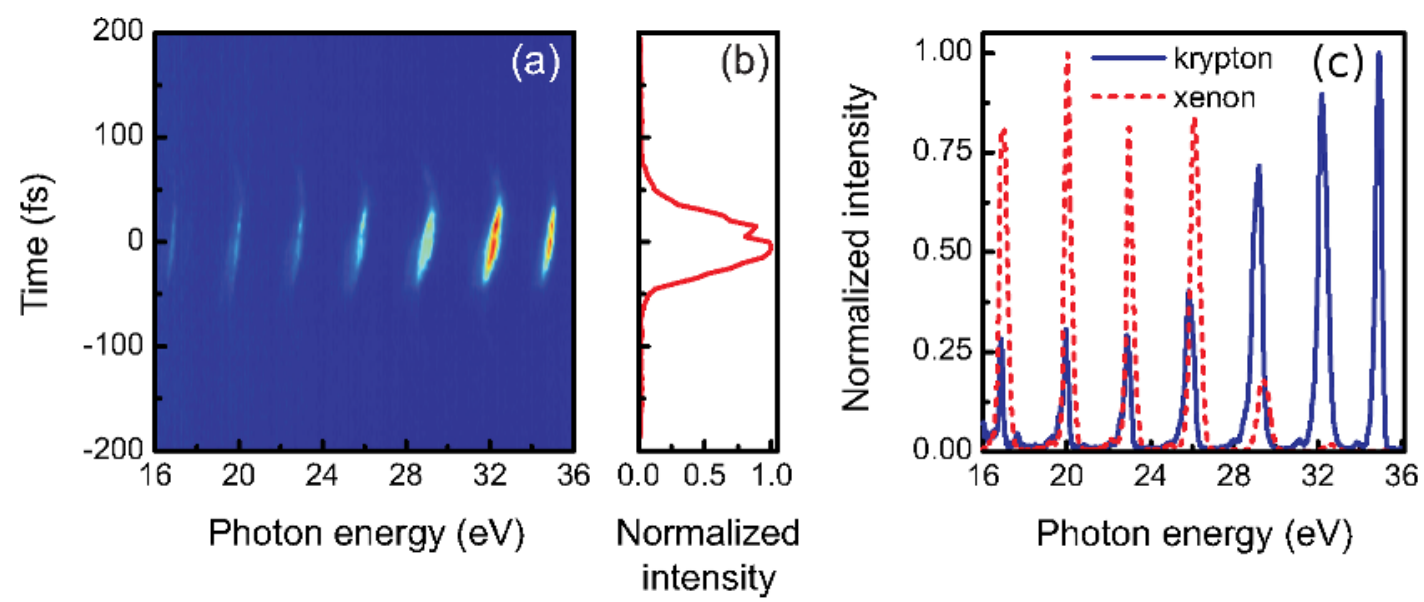

Fig. 2. (a) Scattered harmonics spectra acquired in krypton as a function of photon energy and delay of one laser pulse with respect to the other two (which are kept synchronized). (b) Spectral integral of the harmonic signal reported in panel (a) as a function of the delay. (c) Comparison between scattered harmonic spectra acquired in krypton and xenon at temporal synchronization among the three driving pulses.

signal-to-noise ratio. As expected, the cutoff observed in xenon (red dashed line) is at lower photon energies with respect to krypton; in the latter medium the signal extends well beyond the acquired spectral range as demonstrated by the weak second-oder peaks visible in the spectrum. The slightly different position of the harmonic peaks (in particular a blue-shift of harmonics produced in xenon with respect to krypton, which increases with increasing harmonic order) confirms that the nature of the generating medium plays a non-trivial contribution in the formation of the scattered harmonic emission.

\section{Conclusions}

The harmonic generation process we have considered is deeply in the non-perturbative regime, where the role of microscopic (like the intensity dependence of the atomic dipole phase) and macroscopic effects (like phase matching) play a significant role. In particular, numerical simulations show that the appearance of harmonics not overlapped to the three driving beams cannot be interpreted as XUV radiation scattered from the transient grating produced in the generating medium, but as constructive interference of microscopic nonlinear dipole emissions.

The investigation of high-order harmonic generation by interfering pulses, from both the theoretical and experimental point of view, could pave the way to novel shemes for efficient harmonic generation as well as to novel approaches for background-free high harmonic spectroscopy in atoms and molecules.

\section{References}

1. F. Krausz, M. Ivanov, Rev. Mod. Phys. 81, (2009) 163.

2. A. Rupenyan, J. B. Bertrand, D. M. Villeneuve, and H. J. Wörner, Phys. Rev. Lett. 108, (2012) 033903.

3. J. B. Bertrand, H. J. Wörner, H.-C. Bandulet, É. Bisson, M. Spanner, J.-C. Kieffer, D. M. Villeneuve, and P. B. Corkum, Phys. Rev. Lett. 106, (2011) 023001.

4. J. P. Farrell, L. S. Spector, M. B. Gaarde, B. K. McFarland, P. H. Bucksbaum, and M. Gühr, Opt. Lett. 35, (2010) 2028.

5. G. Sansone, C. Vozzi, S. Stagira, M. Nisoli, Phys. Rev. A 70, (2004) 013411. 